\title{
Contact lens electroretinography in preterm infants from 32 weeks after conception: a development in current methodology
}

\author{
Helen Mactier, Ruth Hamilton, Michael S Bradnam, Thomas L Turner, John Dudgeon
}

\begin{abstract}
Aim-To assess the feasibility of using a contact lens electrode to record the electroretinogram (ERG) in preterm infants less than 35 weeks after conception. Methods-The ERG was recorded from seven very low birthweight preterm infants on a total of 14 occasions using an infant monkey contact lens electrode. Age at recording the first ERG ranged from 23 to 51 days (gestational age 32-34 weeks), and weight ranged upwards from $1100 \mathrm{~g}$.

Results-No complications were observed. With advancing age and maturity the dark adapted rod threshold decreased, indicating increased retinal sensitivity. Conclusions-Contact lens recording of the ERG from extremely small immature preterm infants is a practicable and well tolerated procedure. This method of recording the ERG will enable further evaluation of retinal development in this vulnerable population.
\end{abstract}

(Arch Dis Child Fetal Neonatal Ed 2000;82:F233-F236)

Keywords: electroretinography; preterm; retinopathy of prematurity

It has been known for over 25 years that the electroretinogram (ERG) is detectable within a few hours of birth in all normal infants of at least 34 weeks gestation, provided that a sufficiently bright light stimulus is presented. ${ }^{1}$ More recent studies have shown an ERG as early as 31 weeks after conception, ${ }^{2-4}$ and normative data have been established for healthy well nourished preterm infants approaching term gestation. $^{56}$

The International Society for Clinical Electrophysiology of Vision recommends the use of corneal electrodes for recording the ERG. Of the various corneal electrodes available, the speculum mounted "bipolar" contact lens electrode has the most stable electrical configuration and records the highest amplitude ERG recordings. ${ }^{7}$ Previous studies of the ERG in preterm infants less than 34 weeks after conception have used a conductive silver thread electrode $e^{2-48}$ because the smallest human contact lens electrode currently available (premature infant contact lens electrode; Hansen Laboratories, Iowa City, Iowa, USA) is too large to fit in the eye of the smallest preterm infants. The conductive fibre electrode is well tolerated but does not maintain eyelid opening, a prerequisite for calculation of retinal illuminance. Knowledge of retinal illuminance is required to be able to deduce photoreceptor function from the ERG. ${ }^{9}$

Retinal photoreceptors are both anatomically and functionally immature at birth, even in term infants, ${ }^{10}$ and may be particularly susceptible to nutritional insufficiency and/or damage from retinopathy of prematurity (ROP) in the smallest and most preterm infants. ${ }^{112}$ An improved electroretinographic technique would allow better assessment of retinal function in these immature infants, in whom photoreceptors are at a critical stage of development.

The aims of this study were to establish the feasibility of using a modified contact lens electrode to record the ERG in extremely small preterm infants within the nursery setting, and to assess how well this procedure would be tolerated.

\section{Methods}

Parents of non-dysmorphic infants of 30 weeks gestation or less were approached and asked for consent for inclusion of their newborn infant in the study. The study was approved by the local ethics committee, and informed written consent was obtained from a parent in each case.

All recordings were made in a side room of the special care baby unit in the Queen Mother's Hospital, Yorkhill NHS Trust, Glasgow. Heavy curtains were hung to obtain virtual darkness, and dim red illumination from an A-P darkroom safelight (Photo Plastic, SA, Spain) allowed continuous observation of the infant and operation of equipment. When supplemental oxygen was being administered (11 out of 14 sessions), continuous oxygen saturation monitoring was performed. All babies wore apnoea monitors (MR10, Graseby Medical Ltd, Watford, Middx, UK). Recording sessions were attended by a senior registrar in paediatrics (HM).

To minimise incidence of pupillary dilatation, ERG recordings were scheduled to precede routine ophthalmological screening for ROP. Indirect ophthalmoscopy causes bleaching of photoreceptors ${ }^{13}$ and would certainly affect ERG recordings for some considerable time thereafter.

SUBJECTS

Seven infants were studied on a total of 14 occasions. One ERG series was recorded from each of three infants; the other infants had one or more follow up ERG series recorded at about two week intervals. All infants had required a period of assisted ventilation, ranging from 20 hours to 25 days (mean 10.7 
Table 1 Summary of age, maturity, weight, size of palpebral fissure, pupillary diameter, and retinal findings in preterm infants

\begin{tabular}{|c|c|c|c|c|c|c|c|c|c|}
\hline Infant & Sex & $\begin{array}{l}\text { Gestational age } \\
\text { (weeks) }\end{array}$ & Birth weight (g) & $\begin{array}{l}\text { Age } \\
\text { (days) }\end{array}$ & $\begin{array}{l}\text { Post-conceptional } \\
\text { age (weeks) }\end{array}$ & Weight $(g)$ & $\begin{array}{l}\text { Pupillary } \\
\text { diameter } \\
(\mathrm{mm})\end{array}$ & $\begin{array}{l}\text { Palpebral } \\
\text { fissure }(\mathrm{mm})\end{array}$ & Fundoscopy \\
\hline \multirow[t]{3}{*}{$\overline{\mathrm{A}}$} & $M$ & 27.14 & 600 & 51 & 34.43 & 1100 & 4 & 10 & Stage $1 \mathrm{ROP}$ \\
\hline & & & & 65 & 36.43 & 1600 & 4 & 12 & Stage 2 ROP \\
\hline & & & & 79 & 38.43 & 2000 & 5 & 13 & Stage 3 ROP \\
\hline \multirow[t]{3}{*}{ B } & $\mathrm{F}$ & 28.86 & 1200 & 30 & 33.14 & 1590 & 6 & 10 & Normal \\
\hline & & & & 42 & 34.86 & 2000 & 6 & 12 & Normal \\
\hline & & & & 58 & 37.14 & 2350 & 6 & 13 & Normal \\
\hline $\mathrm{C}$ & $\mathrm{F}$ & 28.57 & 1040 & 27 & 32.43 & 1350 & 7 & 10 & Normal \\
\hline $\mathrm{D}$ & $\mathrm{F}$ & 30.29 & 1300 & 23 & 33.57 & 1620 & 5 & 14 & Normal \\
\hline $\mathrm{E}$ & M & 29.86 & 880 & 41 & 34.71 & 1630 & 5 & 14 & Normal \\
\hline \multirow[t]{2}{*}{$\mathrm{F}$} & $\mathrm{F}$ & 25.43 & 800 & 47 & 32.14 & 1320 & 5 & 14 & Stage 2 ROP \\
\hline & & & & 82 & 37.14 & 2760 & 6 & 14 & Post laser photocoagulation \\
\hline \multirow[t]{3}{*}{ G } & $\mathrm{F}$ & 27.86 & 980 & 38 & 33.29 & 1380 & 4.5 & 10 & Stage 2 ROP \\
\hline & & & & 52 & 35.29 & 1670 & 5 & 12 & Stage 2 ROP \\
\hline & & & & 59 & 36.29 & 2020 & 5 & 13 & Threshold disease \\
\hline
\end{tabular}

ROP, retinopathy of prematurity.

days). Infants were first studied as soon as they had been weaned from ventilatory support and were considered by medical and nursing staff to be tolerant of moderate handling. Gestational age at birth ranged from 25 to 30 weeks and birth weights ranged from 600 to 1300 g. Gestational age was determined from obstetric notes; all dates had been confirmed by early ultrasound screening. In all cases, clinical assessment of gestational age corresponded to maternal notes.

Age at recording of the first ERG ranged from 23 to 51 days (mean 37 days), which corresponded to 32 to 34 weeks after conception. Weight ranged from 1100 to 1630 (mean 1427) g.

Four infants had normal fundi at the time of their first ERG study, one had stage 1, and two had stage 2 ROP. Two infants ( $F$ and $G$ ) subsequently developed threshold $\mathrm{ROP}^{14}$ and were treated with binocular indirect ophthalmoscope diode laser photocoagulation. Infant $F$ had a follow up ERG two weeks after treatment. At outpatient follow up, both infants treated with laser photocoagulation had flat healthy maculae and normal visual fixation.

Table 1 summarises age, maturity, weight, size of palpebral fissure, pupillary diameter, and retinal findings.

PROCEDURE

Shortly after feeding, the infants had both pupils dilated with one drop each of $0.5 \%$ cyclopentolate and $2.5 \%$ phenylephrine. Sterile light occluding patches were then applied to both eyes and the infant was allowed to dark adapt in its cot or incubator for a minimum of 30 minutes. It was then transferred to the darkened side room of the nursery, swaddled, and placed supine in a modified incubator with a customised aperture in the cover to allow accurate and safe positioning of the ganzfeld bowl directly over the infant's face (fig 1). The mattress was padded to support the infant with its eyes $8 \mathrm{~cm}$ from the viewing aperture of the ganzfeld bowl. A guard silver/silver chloride electrode was applied to the forehead, and, under dim red illumination, the eye patches were removed and the dilating protocol repeated. A contact lens electrode was then placed in the right eye using $0.5 \%$ proxymetacaine as local anaesthetic and $2 \%$ hypromellose

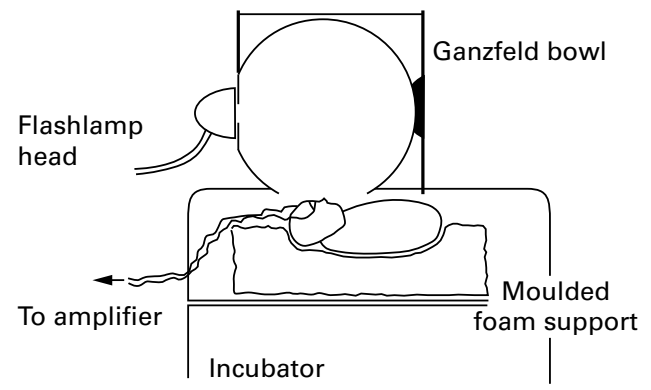

Figure 1 Diagram showing ganzfeld bowl positioned over customised aperture in incubator cover. Infant lies supine as illustrated, with his/her eyes $8 \mathrm{~cm}$ from the aperture of the bowl.

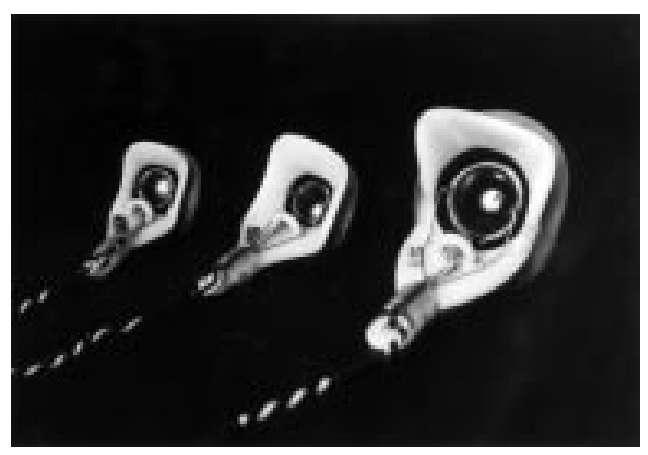

Figure 2 Bipolar Burian-Allen contact lens electrodes. From left to right: infant monkey size 4 (used in this study); conventional premature infant size; adult size.

as a lubricant. An infant monkey size 4 bipolar contact lens electrode (Hansen Laboratories) was used in each case (fig 2).

Stimuli were delivered into the ganzfeld bowl by a Grass photic stimulator PS33 Plus (Astromed Inc; W Warwick, Rhode Island, USA) set at its highest intensity. A selection of calibrated neutral density Wratten filters (Kodak, Hemel Hempstead, Herts, UK) was used to vary the light incident upon the infant's cornea over four log units, from 5.935 to 0.0002 cd.s $/ \mathrm{m}^{2}$.

The signal was differentially amplified and filtered (bandwidth $0.5-100 \mathrm{~Hz}$ at $3 \mathrm{~dB}$ down). This filtered amplified signal was recorded using custom designed software. ${ }^{15}$ The software controlled stimulus delivery, synchronised data acquisition, rejected artefacts, and performed real time signal averaging.

A series of white flashes of progressively increasing intensity was presented at one 
second intervals. Flash intensity was increased in 0.4 or (at brighter intensities) $0.2 \log$ unit steps. Traces were averaged on screen until either an ERG trace was discernible, or the trace was deemed flat (between three and 50 averages). The recording was repeated at each stimulus intensity to examine reproducibility. Each procedure occupied about 45 minutes after dark adaptation.

Our experimental protocol was tested in two healthy adult volunteers. Two different eye positions were tested in random order: face flush with the ganzfeld bowl, as is standard, or face positioned $8 \mathrm{~cm}$ away from the ganzfeld bowl aperture.

INTERPRETATION OF DATA

Where two interpretable traces were obtained at the same stimulus intensity, these were computer averaged.

To avoid making incorrect assumptions of posterior nodal distance and media transmissivity in extremely preterm infants, thresholds are presented in terms of troland values calculated from corneal illuminance and pupil diameter, rather than true retinal illuminance. ${ }^{12} 1617$

The dark adapted rod threshold $(2 \mu \mathrm{V}$ criterion) was derived from each series of ERG recordings by linear regression of b-wave amplitude versus log retinal illuminance, expressed as troland values.

\section{Results}

All recording sessions were completed without incident. No ocular or systemic complications were observed.

Interpretable ERGs were obtained at between seven and 11 light intensities during each recording session. Measurements were repeated at most stimulus intensities, and reproducibility (as judged by two independent observers) was $95 \%$ (155 out of 164 pairs).

Face position made no difference to results obtained from the two adult controls. It was assumed therefore that infants lying with their eye $8 \mathrm{~cm}$ from the ganzfeld bowl aperture received full field stimulation.

THE A-WAVE

At all ages, an a-wave was only discernible at stimulus intensities attenuated by more than 2 $\log$ units of filter $\left(>0.6 \mathrm{~cd} . \mathrm{s} / \mathrm{m}^{2}\right)$. The a-wave latency shortened with increasing stimulus intensity and this applied at all ages (fig 3A).

DARK ADAPTED ROD THRESHOLD

The dark adapted rod threshold was obtained in 11 out of 14 recording sessions (79\%). The dark adapted rod threshold decreased with increasing maturity (fig 3B). After laser photocoagulation in one infant $(F)$, the ERG showed a threshold increase of $0.2 \mathrm{log}$ units. The data obtained from this infant after laser photocoagulation were not included in the regression analysis (fig 3B).

\section{Discussion}

This study shows the practicability of obtaining high quality reproducible ERG recordings using a contact lens electrode from very small
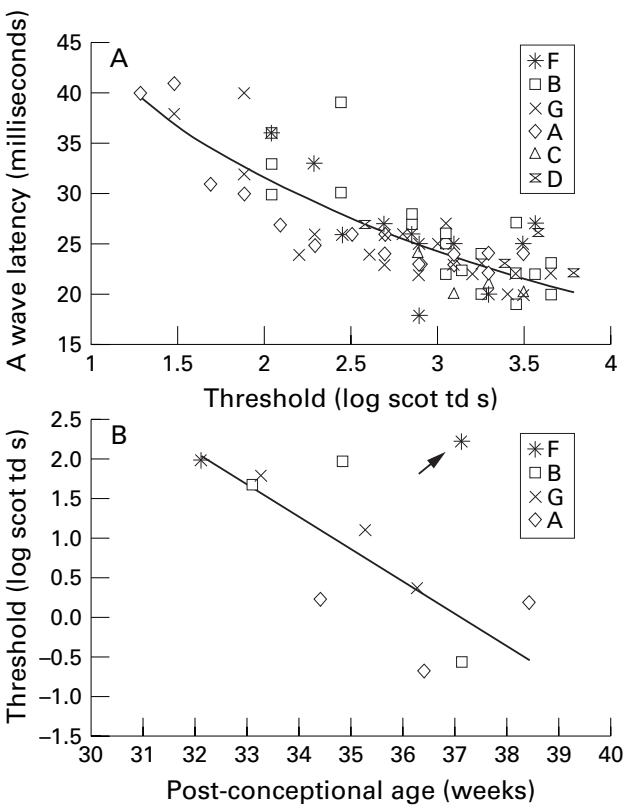

Figure 3 (A) A-wave latency versus troland values. $A$ logarithmic regression curve is fitted to these (non-independent) data to show the trend towards earlier a-waves at brighter retinal illuminances. (B) Dark adapted rod thresholds ( $2 \mu \mathrm{V}$ criteria) were obtained from four of the seven infants. These are plotted against post-conceptional age in weeks. A regression line is fitted to these (non-independent) data to show the trend towards increased rod photoreceptor sensitivity as maturity increases. Infant $F$ underwent laser treatment between ERG recordings. The point after the laser treatment (indicated with an arrow) is not included in the regression analysis. Scot td s, Scotopic troland seconds.

immature preterm infants, within the setting of the neonatal intensive care nursery. The procedure was well tolerated, and indeed, in several instances, recording the ERG was considerably better tolerated than the subsequent routine ophthalmological examination. Most infants slept through the entire recording session. Complications that may follow the use of contact lens electrodes include corneal abrasion or infection; we saw no evidence of the latter. In the absence of slit lamp examination, minor corneal abrasion cannot be excluded, but would in any case be expected to heal spontaneously.

In the most immature infants, the conjunctival sac is too shallow to permit insertion of the speculum of a standard premature infant contact lens electrode (fig 2). The infant monkey electrode was chosen because the external dimensions of its integral speculum are considerably smaller than those of the conventional premature infant contact lens electrode. The diameter of the active electrode, however, is reduced by only $0.2 \mathrm{~mm}(4.3 \%)$.

In infants weighing $>2400 \mathrm{~g}$, the infant monkey electrode tended to be easily dislodged by blinking, and the premature infant electrode was better retained. An exception to this rule of thumb was an infant who had been treated with oral steroids to facilitate weaning from the ventilator. Oral steroids cause fluid retention and periorbital puffiness, rendering insertion of the contact lens electrode more difficult. Because of difficulty in retaining the smaller electrode in 
the eye of larger infants, the two electrodes were not compared in any one infant.

To achieve full field stimulus conditions, the subject's face should normally be positioned flush with the opening of the ganzfeld bowl. This position is impossible to achieve in tiny premature infants, although the infants were positioned as close as possible to the opening of the ganzfeld bowl and both swaddled in and nursed upon white sheets. Our adult data suggest that full field stimulus conditions were met. Baird Mets et al ${ }^{18}$ found a slight reduction in a-b wave amplitude in adult volunteers positioned similarly to our babies compared with standard recording conditions; however, the data were confounded by simultaneous comparison of clear adult and paediatric diffusing electrodes.

The main purpose of this study was to examine the feasibility of using a modified contact lens electrode to record the ERG routinely from infants less than 35 weeks after conception, hence the recording procedure was kept as simple as possible. We have confirmed the findings of Birch et $a \bar{l}$ that rod threshold decreases with advancing maturity, and have extended this observation to infants between 32 and 36 weeks after conception. Adult sensitivity is achieved at around six months after birth in normal infants. ${ }^{19}$

Previous work in preterm infants has shown shortening of a-wave latency, as measured at maximal stimulus intensity, with advancing maturity. ${ }^{23}$ Because of the small number of infants in the present study, it was not possible to confirm this observation. However, we have shown that in preterm infants, as in older subjects, a-wave latency shortens with increasing stimulus intensity. ${ }^{9}{ }^{10}$ Our observation that the a-wave became evident at corneal illuminances of around $0.6 \mathrm{~cd} . \mathrm{s} / \mathrm{m}^{2}$ is in agreement with Baird Mets et al. ${ }^{18}$

To the best of our knowledge, this is the first time that the ERG has been recorded from the same infant both before and after laser treatment for ROP. One study reported an attenuated scotopic ERG at six months of age after laser photo-ablation of the peripheral retina. Using the same recording conditions, the investigators were unable to record the ERG in another four children who had required vitreoretinal surgery for the treatment of stage 5 ROP. ${ }^{20}$ Another study of children aged between six months and nine years who had a history of ROP showed reduced rod b-wave sensitivity in all three children who had been treated with either cryotherapy or laser photo-ablation, and reduced saturated rod b-wave amplitude in two of the three. ${ }^{21}$

Recording the ERG from extremely preterm babies using contact lens electrodes is a safe and practicable procedure. Further evaluation of the use of electroretinography to study rod and cone function in these very vulnerable infants is warranted.

We acknowledge the help of Hansen Laboratories in lending the infant monkey electrode and thank Professor Gordon Dutton for helpful comments. R H was partially funded by a grant from for helpful comments. R H
the Ulverscroft Foundation.

1 Winkelman JE, Horsten GPM. The ERG of premature and full-term born infants during their first days of life. Ophthalmologica 1962;143:92-101.

2 Mactier H, Dexter JD, Hewett JE, Latham CB, Woodruff $\mathrm{CW}$. The electroretinogram in preterm infants. $\mathcal{F}$ Pediatr 1988;113:607-12

3 Leaf AA, Green CR, Esack A, Costeloe KL, Prior PF. Maturation of electroretinograms and visual evoked potentials in preterm infants. Dev Med Child Neurol 1995;37:814-26.

4 Grose J, Harding GFA, Wilton AY, Bissenden JG. The maturation of the pattern reversal VEP and flash ERG in pre-term infants. Clinical Vision Sci 1989;4:239-46.

5 Birch EE, Birch DG, Petrig B, Uauy R. Retinal and cortical function of very low birthweight infants at 36 and 57 function of very low birthweight infants at 36 and 57.

6 Kennedy KA, Ipson MA, Birch DG, et al. Light reduction and the electroretinogram of preterm infants. Arch Dis Child 1997;70:F168-73.

7 Marmor MF, Zrenner E. Standard for clinical electroretinography. Doc Ophthalmol 1999;97:143-56. Text available at http://www.isecv.org/aug/iscev/standards/erg1 999.html.

8 Dawson WW, Trick GL, Litzkow CA. Improved electrode for clinical electroretinography. Invest Ophthalmol Vis Sci 1979;18:988-91.

9 Hood DC, Birch DG. Assessing abnormal rod photoreceptor activity with the a-wave of the electroretinogram: applitor activity with the a-wave of the electroretinogram: app

10 Fulton AB, Hansen RM. The rod sensitivity of dark adapted human infants. Curr Eye Res 1992;11:1193-8.

11 Uauy RD, Birch DG, Birch EE, Tyson JE, Hoffman DR. Effect of dietary omega-3 fatty acids on retinal function of very-low-birth-weight neonates. Pediatr Res 1990;28:48592.

12 Fulton $A B$, Hansen RM. Photoreceptor function in infants and children with a history of mild retinopathy of prematurity. F Opt Soc Am 1996;13:566-71.

13 Bradnam MS, Montgomery DMI, Moseley H, Dutton, GN. Quantitative assessment of the blue-light hazard during indirect ophthalmoscopy and the increase in the "safe" operating period achieved using a yellow lens. Ophthalmoloperating period achieved
ogy 1995;102:799-804.

14 Committee for the Classification of Retinopathy of Prematurity. An international classification of retinopathy of prematurity. Arch Ophthalmol 1984;102:1130-4.

15 Bradnam MS, Evans AL, Montgomery DMI, et al. A personal computer-based visual evoked potential stimulus and recording system. Doc Ophthalmol 1994;86:81-93.

16 Hansen RM, Fulton AB, Harris SJ. Background adaptation in human infants. Vision Res 1986;26:771-9.

17 Makous W. Optics and photometry. In: Carpenter R, Robson J, eds. Vision research. Oxford: Oxford University Press, 1998.

18 Baird Mets M, Smith VC, Pokorny J, Pass A. Postnatal retinal development as measured by the electroretinogram in premature infants. Doc Ophthalmol 1995;90:111-27.

19 Fulton AB. The development of scotopic retinal function in human infants. Doc Ophthalmol 1988;69:101-9.

20 Cherry TA, Lambert SR, Capone A. Electroretinographic findings in stage 5 retinopathy of prematurity after retinal reattachment. Retina 1995;15:21-4.

21 Fulton AB, Hansen RM. Electroretinogram responses and refractive errors in patients with a history of retinopathy of prematurity. Doc Ophthalmol 1996;91:87-100. 